Boise State University

ScholarWorks

Counselor Education Faculty Publications and

Presentations

Department of Counselor Education

4-1-2019

\title{
Continuing Education in Motivational Interviewing for Addiction Counselors: Reducing the Research-to-Practice Gap
}

\author{
Diana M. Doumas \\ Boise State University \\ Raissa M. Miller \\ Boise State University \\ Susan Esp \\ Boise State University
}




\title{
Continuing Education in Motivational Interviewing for Addiction Counselors: Reducing the Research-to-Practice Gap
}

\author{
Diana M. Doumas* \\ Department of Counselor Education \\ Institute for the Study of Behavioral Health and \\ Addiction \\ Boise State University \\ dianadoumas@boisestate.edu
}

\author{
Raissa Miller \\ Department of Counselor Education \\ Institute for the Study of Behavioral Health and \\ Addiction \\ Boise State University
}

\author{
and \\ Susan Esp \\ School of Social Work \\ Institute for the Study of Behavioral Health and Addiction \\ Boise State University
}

\begin{abstract}
Acknowledgments
The authors wish to thank Ben Swanson for assistance in workshop development and delivery and April Johnston and Sherise Porchia for assistance with data management.

Funding for this study was provided in part by a State of Idaho Millennium Fund Grant Senate Bill No. 1144.
\end{abstract}

\begin{abstract}
The researchers evaluated the effectiveness of continuing education training in Motivational Interviewing (MI) for addiction counselors. Participants reported a significant increase in counseling self-efficacy and 86.8\% reported increasing MI use in their practice. Only 3.9\%, however, participated in post-training consultation.
\end{abstract}

Keywords: motivational interviewing, continuing education, training workshop, self-efficacy

Among individuals in the United States aged 12 or older, 15.7 million are diagnosed with an alcohol use disorder and 7.7 million are diagnosed with an illicit drug use disorder (Substance Abuse and Mental Health Services Administration [SAMHSA], 2017). Among those, only 4.4\% and $11.0 \%$ with an alcohol use disorder or illicit substance use disorder, respectively, received treatment for their disorder (SAMHSA, 2017). As the problem of addiction grows in the United States, the need for professionals who are competent in treating substance-related disorders is also growing. This need is supported by estimates from the U.S. Bureau of Labor Statistics projecting a $22 \%$ increase in the need for substance abuse and behavioral disorders counselors between the years of 2014 to 2024 (U.S. Bureau of Labor Statistics, 2015).

\section{Addiction Counseling Training}

In 2009, the Council for Accreditation of Counseling and Related Educational Programs (CACREP) added addictions counseling to the CACREP program options (CACREP, 2009). The 2009 accreditation standards also included the development of addiction competencies for other specialty tracks, as well as the integration of addiction education into the common core curricular experiences (Lee, Craig, Fetherson, \& Simpson, 2013; Moro, Wahesh, Likis-Werle, \& Smith, 2016). Prior to the transition to 2009 accreditation standards, graduates from CACREP accredited programs may not have received any education in addiction counseling. Thus, a large percentage of practicing professional counselors may not have received training in addiction-related issues during their graduate program, creating a need for professional training in this area. 
This is an author-produced, peer-reviewed version of this article. The final, definitive version of this document can be found online at Journal of Addictions \& Offender Counseling, published by American Counseling Association. Copyright restrictions may apply. doi: 10.1002/jaoc.12055

In addition to professional counselors trained for licensure through CACREP accredited programs, many states and several national organizations offer licensure and/or certification in addictions counseling to a diverse group of professionals with a variety of educational experiences and academic degrees (Kerwin, Walker-Smith, \& Kirby, 2006). Nationally, however, the education and training required for addiction counseling lacks uniformity (Chasek \& Kawata, 2016), with large variations in requirements and standards for licensure and certification (Astramovich \& Hoskins, 2013). Although standards for practice suggest that health care professionals, including addiction counselors, are expected to provide evidence-based, scientifically supported treatment (Miller, Sorensen, Selzer, \& Brigham, 2006), research indicates that most addiction counselors are not prepared to provide evidence-based practices (EBPs) when they enter the field (Weissman et al., 2006). Thus, professional training, including skills-based training, is needed to promote the integration of EBPs into addiction counseling practice.

\section{The Gap Between Research and Practice}

Meta-analyses and reviews of the research identify several empirically supported treatments for substance use disorders including contingency management (CM; Griffith, Rowan-Szal, Roark, \& Simpson, 2000), cognitivebehavioral therapy (CBT; Irvin, Bowers, Dunn, \& Wang, 1999), and motivational interviewing (MI; Dunn, Deroo, \& Rivara, 2001).

Although the literature supports the efficacy of EBPs for treating substance use disorders, a significant gap remains between research and clinical practice in substance abuse programs (Evans, Koch, Brady, Meszaros, \& Sadler, 2013; Miller et al., 2006). National interview data with 340 substance abuse treatment center directors indicates that although $90 \%$ of these treatment centers use CBT, only 55\% use MI and only 30\% use CM (Olmstead, Abraham, Martino, \& Roman, 2012). These data suggest that although there is an increasing need for the use of EBPs substance abuse treatment, EBPs continue to be underutilized in substance abuse treatment centers.

One explanation for the research-practice gap is that there are significant barriers that reduce the probability that EBPs will be implemented in substance abuse treatment programs. Researchers have identified several therapist and organizational variables that may decrease the adoption of EBPs by addiction counselors. For example, addiction counselors may not have adequate time to learn new therapies (Campbell, Catlin, \& Melchert, 2003; Miller et al., 2006). Additionally, lack of basic knowledge and skills may pose barriers to the implementation of EBPs into practice (Haug et al., 2008) and training opportunities may not be available to clinicians (Weissman et al., 2006). Further, procedures used for training EBPs in efficacy trials may not translate to real world settings (Glasglow, Lichtenstein, \& Marcus, 2003), limiting the utility of this research to provide guidance for successful dissemination of EBPs into addiction counseling practice.

\section{Motivational Interviewing}

Research indicates that addiction counselors are open to adopting EBPs (Haug, Shopshire, Tajima, Gruber, \& Guydish, 2008) and among EBPs, addiction counselors are most ready to adopt MI (McGovern, Fox, Xie, \& Drake, 2004). Because MI has gained momentum as an EBP for substance abuse, demands for professional training in MI have increased (Schumacher, Madson, \& Norquist, 2011). MI is a non-judgmental, client-centered, yet directive treatment approach that is designed to elicit positive behavioral change through exploring and resolving client ambivalence (Miller \& Rollnick, 2013). A central component of MI is providing individualized feedback to clients about their alcohol use and associated risk. Because MI can be implemented as a brief evidence-based intervention, MI is consistent with managed care and insurance models emphasizing brief empirically supported treatments. Further, because MI combines both person-centered and directive approaches, MI has the capacity to appeal to counselors from a broad range of theoretical orientations, enhancing potential post-training adoption of MI into addiction counseling practice.

Results from meta-analyses on brief interventions for alcohol use disorders support the efficacy of MI in the treatment of excessive alcohol use (Vasilaki, Hosier, \& Cox, 2006) and is as efficacious as extended treatment conditions for alcohol use disorders (Moyer, Finney, Swearingen, \& Vergun, 2002). More recent systematic reviews and metaanalyses of the research also support the efficacy of MI (Lenz, Rosenbaum, \& Sheperis, 2016; Lundahl et al., 2013; Riper et al., 2014; Sayegh, Huey, Zara, \& Jhaveri, 2017; VanBuskirk \& Wetherell, 2014). Findings indicate MI delivered in medical settings is effective in reducing in substance use (Lundahl et al., 2013; VanBuskirk \& Wetherell, 
This is an author-produced, peer-reviewed version of this article. The final, definitive version of this document can be found online at Journal of Addictions \& Offender Counseling, published by American Counseling Association. Copyright restrictions may apply. doi: 10.1002/jaoc.12055

2014); MI is effective in the treatment of co-morbid alcohol use disorders and depression (Riper et al., 2014); and MI promotes reductions in use across a range of substances (Lenz et al., 2016), even several months after the implementation of the intervention (Sayegh et al., 2017).

\section{Solutions to Address the Research-Practice Gap}

Self-study materials and didactic training workshops are two common strategies for bridging the research-practice gap (Martino et al., 2011). Self-study typically includes reading the MI literature and treatment manuals, whereas workshops typically include didactic presentations, demonstrations, and interactive exercises delivered over a period of 1-3 days (Schwalbe, Oh, \& Zweben, 2014). Results from a recent meta-analysis indicate MI training workshops are more efficacious in developing MI skills than self-study programs (Schwalbe et al., 2014). The authors also note there were no differences between standard workshops and workshops that included innovative elements such as feedback on audio-recorded client sessions or group follow-up booster session, although post-workshop feedback and coaching were both efficacious in sustaining gains in MI skills. Similarly, results from two earlier systematic reviews and a meta-analyses indicate MI skills increase from pre-training to post-training workshops and that skills in MI implementation are enhanced through post-workshop feedback and coaching (Barwick, Bennett, Johnson, McGowen, \& Moore, 2012; de Roten, Zimmermann, Ortega, \& Delpland, 2013; Madson, Loignon, \& Lane, 2009).

\section{Counselor Self-Efficacy}

In addition to acquiring knowledge and skills, successful integration of EBPs is also related to counselor self-efficacy. According to Bandura (1986), self-efficacy is the belief in one's ability to complete a task, involving an individual's cognitive, social, and behavioral skills. Larson and Daniels (1998) applied this concept to counseling, suggesting counseling self-efficacy is one's beliefs or judgments about his or her ability to effectively counsel clients. Research indicates that counseling self-efficacy can be increased through opportunities that allow practice and mastery of skills (Greason \& Cashwell, 2009). Competency and counseling self-efficacy can be built through role-plays and feedback (Daniels \& Larson, 2001). These experiential training activities are consistent with those provided in MI workshops (Schwalbe et al., 2014), suggesting MI workshops that include role-plays with practice feedback are likely to positively impact counselor self-efficacy.

Research indicates counseling self-efficacy is associated with positive client outcomes (Reese et al., 2009; Urbani et al., 2002). Additionally, counseling self-efficacy an important factor in the likelihood of counselors using specific counseling skills (Iarussi, Tyler, Littlebear, Hinkle, 2013). For example, recent research indicates that post-training, counselor self-efficacy is associated with quality of practice, knowledge of EBPs, and use of EBPs (Schiele, Weist, Youngstrom, Stephan, Lever, 2014). Therefore, increasing counselor self-efficacy is an important component of trainings intended to promote practitioner implementation of MI with clients post-training.

\section{Post-Training Consultation and Use of MI Counseling Practice}

Although there is a large body of literature examining the efficacy of methods of dissemination (e.g., self-study, standard workshops, enhanced workshops) on counselors' ability to implement MI, we could find no study examining the effectiveness of MI training on counselor participation in post-training consultation or use of MI in their practice post-training. Although efficacy and effectiveness studies are both important, these study designs serve distinct purposes (Singal, Higgins, \& Waljee, 2014). Specifically, efficacy trials are designed to evaluate outcomes under ideal circumstances, whereas effectiveness studies are designed to evaluate outcomes in the real world setting (Singal et al., 2014).

The majority of studies contained in the systematic reviews and meta-analyses in the literature were efficacy trials, concerned with tracking skill acquisition through a research protocol including coding recorded sessions with actual clients to measure trainees' skills. Although trainees continued to use MI across follow-up periods, it is unclear if trainees would participate in post-training coaching and feedback in the absence of incentives or have used MI in their counseling practice if they were not being followed in this way (e.g., providing recorded session to researchers. Thus, efficacy trials studying the maintenance of MI skills overtime do not provide information on whether or not counselors would typically participate in post-training consultation sessions or chose to use MI in their counseling practice after participating in a training workshop. 
This is an author-produced, peer-reviewed version of this article. The final, definitive version of this document can be found online at Journal of Addictions \& Offender Counseling, published by American Counseling Association. Copyright restrictions may apply. doi: 10.1002/jaoc.12055

\section{The Current Study}

The purpose of this study is to examine the effectiveness of MI training workshops on counselor self-efficacy, selfreported post-training use of MI in counselor practice, and participation in post-training consultation. Prior efficacy studies have examined MI skill acquisition through the monitoring of recorded sessions as part of the research protocol. However, it is unclear if workshop participants would use MI in their counseling practice or participate in post-training coaching in the absence of an experimental monitoring protocol. Because primary barriers to implementing EBPs include not having enough time to learn new therapies (Miller et al., 2006), and lack of training opportunities (Weissman et al., 2006), we were interested in the amount of training needed to impact counselor selfefficacy and to promote the use of MI in addiction counseling practice. We were also interested in what percent of participants would participate in post-training consultation sessions in the absence of incentives.

To achieve this aim, we compared outcomes among training participants who attended either a one-day MI training workshop or a two-day training workshop. We used a quasi-experimental design in which participants elected to participate in either a one-day or two-day workshop. We also invited participants who attended the two-day workshop to participate in up to five free in-person post-training consultation and tracked the number of participants who attended at least one session.

The research questions were: a) Do participants attending a one-day or two-day MI workshop report an increase in self-efficacy from baseline to a 6-week follow-up, b) Do participants attending a one-day or two-day MI workshop report using MI in their addiction counseling practice at a 6-week follow-up? c) Is length of training a moderator of training effects such that there is a difference in outcomes between the one-day and two-day workshops? and d) what percent of workshop participants attended post-training consultation?

\section{Method}

\section{Research Design}

We used a quasi-experimental design comparing one-day workshop participants $(n=58)$ to two-day workshop participants $(n=85)$. We assessed increases in counselor self-efficacy from baseline to the 6-week follow-up and self-reported use of MI at the 6-week follow-up. We examined workshop type (one-day vs two-day) as a moderator of training effects. We also tracked the number of participants attending post-training consultation.

\section{$\underline{\text { Participants }}$}

We recruited professionals working with clients with substance abuse from a series of MI training workshops offered at a public university in an urban area in the Northwest. A total of 143 participants (69.1\% female, 30.9\% male) consented to participate in the study. Ages ranged from 21-69 $(M=41.18, S D=11.48)$. The majority of the sample was White (85.9\%), with 6.3\% Hispanic, 4.9\% Native American, 1.0\% Asian American, and 1.8\% other. The majority of the participants identified their professional identity as counseling (53.2\%), with $17.5 \%$ identifying as social work, $15.4 \%$ as substance abuse counseling, 3.5\% nursing $2.1 \%$ corrections, and $8.3 \%$ other. Years of professional experience ranges from 1-39 $(M=6.17, S D=7.21)$. Overall, $56 \%(n=80)$ of the 143 participants completed the 6week follow-up assessments. We found no differences in any demographics gender, $\chi^{2}(1)=0.35, p=.55$, age, $t(137)$ $=1.75, p=.08$, ethnicity, $\chi^{2}(4)=5.00, p=.29$, or years in practice, $t(137)=156, p=.12$, between those who completed the follow-up assessment and those who did not. There were no differences in the rate of attrition across the one-day and two-day workshop participants, $\chi^{2}(1)=0.96, p=.33$.

\section{Procedure}

We advertised the workshops via email through various state-wide listserve and email databases. Registrants were given the choice to enroll in either a one-day MI workshop (Friday) or a two-day MI workshop (Friday and Saturday). We also offered up to five post-training consultation sessions to participants who attended the two-day workshop. We provided six weekend workshops throughout the year. We recruited study participants from the pool of addiction practitioners enrolled in the training workshops. A member of the research team collected informed consent prior to the start of the training, collected baseline surveys, and provided a unique personal identification number (PIN) to participants to maintain confidentiality. All participants completed the baseline survey immediately prior to the Friday 
workshop and a 6-week online follow-up survey distributed to study participants via email. All surveys took approximately 15 minutes to complete. No incentives were provided for study participation. All study procedures were approved by the University Institutional Review Board.

\section{Training Workshops}

The workshop instructors were faculty at the university offering the training. The lead author selected these instructors due to their extensive experience providing professional trainings and coursework in MI. Each instructor provided the both the Friday and Saturday MI workshops on three weekends. We describe the content of the workshops below.

Friday Workshop. The instructor covered the following topics during the 6 hour training (a) the history of MI and MI as an evidence based practice in treating behavioral health issues, (b) the transtheoretical model and stages of change, (c) the underlying "spirit" of MI, including autonomy, collaboration, evocation, acceptance and compassion, (d) MI principles of RULEs (e.g., resisting the righting reflex, understanding client's motivation, listening to your client and empowering your client, (e) OARS enhancement skills (e.g., open-ended questions, affirmations, reflections and summaries), (f) "traps” (e.g, question/answer trap, premature focus trap, confrontation trap, blaming trap, expert trap, and labeling trap, (g) eliciting change talk and listening for change talk, (h) recognizing and working with client resistance, and (i) recognizing and working with client ambivalence.

Saturday Workshop. The instructor covered the following topics during the 6 hour training (a) developing discrepancies between clients behavior and goals by exploring ambivalence and exploring motivation (Phase I) and strengthening commitment (Phase 2) utilizing MI, (b) elements of change talk (e.g., content, recognition, specific target behavior, and present tense language), (c) preparatory (DARN) and mobilizing language (C) (e.g, desire, ability, reason, need, commitment and taking steps), (d) directive MI skills including asking evocative questions, elaboration, using extremes, looking back, looking forward, exploring goals, assessing feedback, and the readiness ruler, (e) working with resistance (e.g., double-sided or amplified reflection, shifting focus, coming alongside, agreement with a twist, emphasizing personal control and choice, and paradox, and (f) collaborating with clients in creating a change plan (e.g., set goals, offer a menu of change options, arrive at a plan, elicit a commitment, lower barriers to action, and enlist social support).

Workshop Enhancements. The instructor used videos, demonstrations, and role-plays during both the Friday and Saturday workshops to enhance the didactic, lecture material. Additionally, during the Saturday training workshops, participants completed role-plays in groups of three (e.g. counselor, client, and observer). The observer used a worksheet provided by the trainer to rate skills and provided feedback to the participant acting as counselor.

Post-Training Consultation. The third author provided in-person post-training consultation which consisted of up to five 50-minute meetings comprised of case discussion and review of recorded material.

\section{$\underline{\text { Measures }}$}

Demographics. A brief demographic questionnaire designed for this study included basic participant characteristics (e.g., age, gender, race/ethnicity, profession).

Counselor Self-Efficacy. We measured counselor self-efficacy at baseline and the 6-week follow-up with the Substance Abuse Treatment Self-Efficacy Scale (SATSES; Kranz \& O’Hare, 2006). The SATSES is a 32-item, fivefactor scale modified from the Alcohol and Other Drug Self-Efficacy Scale (AODSES; Kranz, 2003). Each item is scored on a 5-point scale ranging from 1 (Very Low) to 5 (Very High). Items load on one of 5 subscales: (a) Assessment and Treatment Planning (10 items), (b) Individual Counseling (7 items), (c) Case Management (6 items), (d) Ethics (5 items), and (e) Group Counseling (4 items).

For the purpose of this study, we used the Assessment and Treatment Planning, Individual Counseling, and Case Management scales. We did not use the Ethics or Group Counseling Scales as the trainings did not address these topics. Example items from the Assessment and Treatment Planning Scale include "How confident are you in determining the client's readiness for treatment/change?," "How confident are you in explaining assessment findings to the client?" Example items from the Individual Counseling Scale include "How confident are you in facilitating the client's engagement in the treatment/recovery process?," and "How confident are you in encouraging and reinforcing all client actions that are determined to be beneficial in progressing toward treatment goals?,” How 
This is an author-produced, peer-reviewed version of this article. The final, definitive version of this document can be found online at Journal of Addictions \& Offender Counseling, published by American Counseling Association. Copyright restrictions may apply. doi: 10.1002/jaoc.12055

confident are you in establishing a helping relationship with the client characterized by warmth, respect, genuineness, concreteness and empathy?” Example items from the Case Management Scale include "How confident are you in continuously assessing and evaluating referral resources to determine their appropriateness?" and "How confident are you in initiating collaboration with referral sources?”

The SATSES has demonstrated reliability including high levels of internal consistency for all subscales ranging from $\alpha=.89-.96$ (Kranz \& O'Hare, 2006). Results of a study further validating the SATSES revealed strong support for the reliability and factorial validity of the scale (Kranz \& O'Hare, 2011). Cronbach's alphas for the current sample were $\alpha=.95, .91$, and $\alpha=.92$ for the Assessment and Treatment Planning, Individual Counseling, and Case Management scales, respectively.

Self-Reported Use of MI. We measured self-reported use of MI at the 6-week follow-up with the following item created for this study: Did you increase your use of MI as a result of the training? We also asked participants to rate their belief in the statement "Other mental health practitioners should learn MI" on a 5-point scale ranging from 1 (Strongly Disagree) to 5 (Strongly Agree).

Participation in Post-Training Consultation. We measured participation in post-training consultation by the number of participants who attended at least one consultation session.

\section{Power Analysis}

We conducted an a priori power analysis using the G*Power 3.1.3 program (Faul, Erdfelder, Lang, \& Buchner, 2007) for a GLM repeated-measures analysis (ANOVA) with two time points. Results of the power analysis indicated a sample size of 54 is needed for power of $\geq 0.95$ to detect a medium effect size of . 25 for the 2-way interaction effect of Time $x$ Group with an alpha level of .05 . Thus, our 6-week sample size of 80 is greater than the sample size needed to provide adequate power for our analyses.

\section{$\underline{\text { Statistical Analysis }}$}

We conducted all analyses using SPSS version 24.0. Prior to analysis, we examined all variables for extreme cases and for normality at baseline and follow-up assessments. We confirmed that participants in the two conditions (oneday; two-day) were equivalent with respect to demographics and baseline outcomes with $t$-tests for continuous variables and chi-square tests for categorical variables. To assess changes in self-efficacy and the moderating effects of training condition, we conducted three GLM repeated-measures analysis of variance (ANOVA) with fixed effects of Time (baseline; 6-week follow-up) and Condition (one-day; two-day). To assess self-reported use of MI at the 6week follow-up and the moderating effect of condition (one-day; two-day), we conducted chi square analyses. To assess the rate of participation in post-training consultation, we calculated the percentage of participants who attended at least one session. We calculated effect size using partial eta squared $\left(\eta^{2}\right)$ for ANOVA analyses, with .01 considered small, .06 considered medium, and .14 considered large (Cohen, 1969; Richardson, 2011). We set the significance level at $p<.05$. We controlled for Type 1 error by using the Holm-Bonferroni procedure (Holm 1979). We selected this method as it corrects for Type I error as effectively as the traditional Bonferroni procedure, but retains more statistical power (Bender \& Lange, 2001; Eichstaedt, Kovatch, \& Maroof, 2013; Wright, 1992).

\section{Results}

\section{Preliminary Analyses}

We found no outliers and all variables were within the normal range for skew and kurtosis. We found no significant differences between participants in the one-day and two-day conditions with respect to gender, $\chi^{2}(1)=2.24, p=14$, ethnicity, $\chi^{2}(4)=8.76, p=.08$, age, $t(141)=-1.55, p=.12$, years in practice, $t(141)=-1.67, p=.10$, or highest degree attained, $\chi^{2}(3)=3.78, p=.29$.

\section{$\underline{\text { Self-Efficacy }}$}

Means, standard deviations, and contrasts for counselor self-efficacy at baseline and the 6-week follow-up are presented in Table 1. Results of the GLM repeated measures ANOVAs indicated a significant main effect for Time but not for the Time x Group interaction effect for each scale. Results indicate counselor self-efficacy increased 
This is an author-produced, peer-reviewed version of this article. The final, definitive version of this document can be found online at Journal of Addictions \& Offender Counseling, published by American Counseling Association. Copyright restrictions may apply. doi: 10.1002/jaoc.12055

significantly from baseline to the 6-week follow-up for the Assessment and Treatment Planning, Individual Counseling, and Case Management scales and there were no differences between participants in the one-day and twoday conditions. The effect size for the main effect for Time was in the medium range.

\section{Self-Reported Use of MI in Counseling Practice}

At a 6-week follow-up, 82.6\% of participants in the one-day training condition reported an increase in the use of MI and $93.5 \%$ of participants in the two-day training condition reported an increase in the use of MI. Results of a chi square analysis indicated no statistically significant difference between the two conditions, $\chi^{2}(1)=2.00, p=.16$. Additionally, $94.7 \%$ and $100 \%$ of the participants in the one-day training and two-day training conditions, respectively, reported the belief that other mental health practitioners should learn MI. Results of a chi square analysis indicated no statistically significant difference between the two conditions, $\chi^{2}(1)=3.70, p=.16$.

\section{Participation in Post-Training Consultation}

Of the 52 participants who attended the two-day workshop, although $7.7 \%(n=4)$ signed up for post-training coaching, only 3.9\% $(n=2)$ attended any post-training consultation sessions. Informal feedback from participants indicated reasons for not attending post-training consultation included not enough time to participate, difficulty scheduling, and problems related to recording clients.

\section{Discussion}

The purpose of this study was to examine the effectiveness of MI continuing education on post-training counselor self-efficacy and self-reported use of MI in addiction counseling practice. We were also interested in the amount of training needed to promote self-efficacy and the use of MI at a 6-week follow-up, as well as rates of participation in post-training consultation without research incentives. Overall, findings supported the impact of the training workshops on increasing counselor self-efficacy and the self-reported increase in the use of MI. There were, however, no differences in self-efficacy or self-reported use of MI in practice between those who attended the one-day training workshop and those who attended the two-day training workshops. Further, only 3/9\% $(n=2)$ workshop participants attending post-training consultation session.

Results from the current study indicated that participants reported an increase in counselor self-efficacy in the areas of assessment and treatment planning, individual counseling, and case management. These results are consistent with research indicating counselor self-efficacy can be built through role-plays and feedback (Daniels \& Larson, 2001) such as those provided in effective MI workshops (Schwalbe et al., 2014). This finding is particularly important because counselor self-efficacy is associated with both the use of EBPs (Schiele et al., 2014), as well as the use of specific counseling skills (Iarussi et al., 2013). Thus, increasing counselor self-efficacy is an important outcome for trainings intended to promote addiction practitioner adoption of EBPs in their counseling practice.

Findings of this study also indicated that at a 6-week follow-up, approximately $90 \%$ of participants reported an increase in the use of MI and nearly 100\% reported that other mental health practitioners should learn MI. Although research suggests that MI training workshops are effective in developing MI skills (Barwick et al., 2012; de Roten et al., 2013; Madson et al., 2009; Schwalbe et al., 2014), the majority of research examining dissemination methods concerns counselors' ability to implement MI with fidelity. To our knowledge, this is the first study examining the effectiveness of MI trainings on counselor report of an increase in the use of MI in their practice in the absence of having their skills tracked and supervised by researchers. Results from this study suggest that a 1-2 day MI training workshop does impact the self-reported use of MI in counseling practice and belief that other practitioners should learn MI.

We did not find evidence for moderating effects of workshop condition. Results revealed no differences between participants attending the one-day workshop and those attending the two-day workshop in increases in counselor selfefficacy and self-reported use of MI in their counseling practice. This finding suggests that the content provided in the first day of training (e.g., transtheoretical model overview, OARS, eliciting change talk, and working with client ambivalence) may be sufficient to increase feelings of self-efficacy in assessment and treatment planning, individual counseling, and case management and an interest in using MI in counseling practice. 
This is an author-produced, peer-reviewed version of this article. The final, definitive version of this document can be found online at Journal of Addictions \& Offender Counseling, published by American Counseling Association. Copyright restrictions may apply. doi: 10.1002/jaoc.12055

Finally, although we offered free post-training consultation to participants who completed the two-day training, only 7.7\% $(n=4)$ of participants signed up for post-training consultation and only 3.9\% $(n=2)$ attended post-training consultation. Informal data collected from participants suggests that they were not able to find the time to participate, they found it too difficult to schedule the sessions, or they had problems with recording client sessions. This finding is consistent with research indicating one of the primary barrier to addressing the research-practice gap is lack of time to learn new therapies (Campbell et al., 2003; Miller et al., 2006) and that findings from efficacy trails do not always generalize to real world conditions (Glasglow et al., 2003).

\section{Limitations and Directions for Future Research}

Although this study contributes to the literature on the effectiveness of MI continuing education training for addiction counselors, certain limitations should be considered. First, the sample was predominantly Caucasian, limiting the generalizability of the results. Future research with more diverse samples is needed. Next, the rate of attrition at the 6-week follow-up (44\%) was high. Although we found no differences in among participants who were retained and who dropped out of the study, it is possible that those who were retained may not have been representative of the full sample in ways that we did not measure. Future researchers should provide incentives for participation to increase participant retention. Next, although both the one-day and two-day workshop participants reported an increase in selfefficacy and self-reported use of MI in counseling practice, we did not measure proficiency in MI skill acquisition. Thus, the reader should not interpret the current findings as suggesting that MI skills can be acquired in a one-day or two-day training. A final limitation of this study is the quasi-experimental design. Although we were not able to randomly assign participants to either the one-day or two-day training conditions, our analyses suggest that there were no differences between participants in the two groups on gender, ethnicity, age, years in practice, or highest degree attained. However, there may be other differences that we did not measures, including prior training or use of MI, which may have impacted the findings of this study. Future research utilizing random assignment to workshop condition would add to the validity of the results of this study.

\section{$\underline{\text { Implications for Addiction Counselors and Agencies }}$}

Findings from this study suggest that even a brief MI training workshop can have a positive impact on self-efficacy and self-reported use of MI in one's counseling practice. Because an extensive literature indicates that MI is effective for treating substance use disorders, it is important that addiction counselors who did not learn MI as part of their education seek out opportunities to learn this important therapy. Addiction counselors can seek out MI training workshops to gain valuable knowledge and skills that they can use with their clients. Although a one-day workshop can have positive effects, research indicates that skills can be enhanced through participating in post-training feedback and coaching. Thus, addiction counselors should seek out trainings that have follow-up feedback and coaching opportunities or seek other consultation if not offered through the training workshop. Because time is a barrier to participating in both workshops and post-training activities that enhance skill acquisition, addiction counselors can talk to their employers to request time to attend trainings and post-training coaching. Addiction counselors may need to explain the values of learning MI for the agency or treatment setting and provide information to their employers about the importance of post-training consultation. Addiction counselors can also ask their employers to arrange agency-wide MI trainings with follow-up feedback and coaching sessions for the entire staff.

Treatment center and agency directors can facilitate the use of MI by addiction counselors by supporting brief trainings followed by feedback and coaching. Because skills in MI implementation are enhanced through post-workshop feedback and coaching, it is important to find ways to incentivize counselors and remove barriers to attending trainings and post-training coaching. Agencies can offer incentives such as training time off with pay, bonuses for participating in post-training coaching sessions, or providing post-training coaching on site. Agencies that have a staff member who is proficient in MI and trained in supervision may also set up an infrastructure so that that staff member can provide coaching and feedback with other staff members who have attended an MI workshop. Additionally, educators trained in MI can offer brief training workshops with follow-up consultation for addiction counselors in their communities. Because addiction counselors typically need continuing education for renewal of certification and licensure, these trainings offer an opportunity to bridge the research-practice gap and increase the use of MI by addiction counselors. 


\section{Conclusion}

The aim of this study was to examine the effectiveness of one-day and two-day continuing education workshops. Findings indicate participants attending either the one-day or two-day continuing education workshop reported increases in counselor self-efficacy and self-reported use of MI in their counseling practice. We also found that although participants were offered post-training consultation, very few participants took advantage of this opportunity. This study adds to the literature identifying ways to promote the use of MI in addiction counseling practice, thereby addressing the research-practice gap.

\section{References}

Astramovich, R. L., \& Hoskins, W. J. (2013). Evaluating addictions counseling programs: Promoting best practices, accountability, and advocacy. Journal of Addictions \& Offender Counseling, 34, 114-124. doi:10.1002/j.2161-1874.2013.00019.x

Bandura, A. (1986). The explanatory and predictive scope of self-efficacy theory. Journal of Social and Clinical Psychology, 4, 359-373. doi:10.1521/jscp.1986.4.3.359

Barwick, M. A., Bennett, L. M., Johnson, S. N., McGowan, J., \& Moore, J. E. (2012). Training health and mental health professionals in motivational interviewing: A systematic review. Children and Youth Services Review, 34(9), 1786-1795. doi:10.1016/j.childyouth.2012.05.012

Bender, R., \& Lange, S. (2001). Adjusting for multiple testing - when and how? Journal of Clinical Epidemiology, 54, 343-349.

Campbell, T. C., Catlin, L. A., \& Melchert, T. P. (2003). Alcohol and other drug abuse counselors' attitudes and resources for integrating research in practice. Journal of Drug Education, 33, 307-323. doi:10.2190/VUHN-X36D-KH56-2P77

Chasek, C. L., \& Kawata, R. (2016). An examination of xducational and training requirements in addiction counseling. Ideas and Research You Can Use: Vistas.

Cohen, J. (1969). Statistical power analysis for the behavioural sciences. New York: Academic Press.

Council for Accreditation of Counseling and Related Educational Programs. (2009). CACREP accreditation manual. Alexandria, VA: Author.

Daniels, J. A., \& Larson, L. M. (2001). The impact of performance feedback on counseling self-efficacy and counselor anxiety. Counselor Education and Supervision, 41, 120-130. doi: 10.1002/j.15566978.2001.tb01276.x

Dunn, C., Deroo, L., \& Rivara, F. P. (2001). The use of brief interventions adapted from motivational interviewing across behavioral domains: a systematic review. Addiction, 96, 1725-1742. doi:10.1046/j.13600443.2001.961217253.x

Eichstaedt, K. E., Kovatch, K., \& Maroof, D. A. (2013). A less conservative method to adjust for familywise error rate in neuropsychological research: the Holm's sequential Bonferroni procedure. NeuroRehabilitation, 32, 693-696. doi:10l3233?NRE-130893

Evans, S. W., Koch, R. J., Brady, C., Meszaros, P., \& Sadler, J. (2013). Community and school mental health professionals' knowledge and use of evidence based substance use prevention programs. Administration and Policy in Mental Health, 40, 319-330. doi:10.1007/s10488-012-0422-z.

Faul, F., Erdfelder, E., Lang, A. G., \& Buchner, A. (2007). G* Power 3: A flexible statistical power analysis program for the social, behavioral, and biomedical sciences. Behavior Research Methods, 39(2), 175-191.

Glasgow, R. E., Lichtenstein, E., \& Marcus, A. C. (2003). Why don't we see more translation of health promotion research to practice? Rethinking the efficacy-to-effectiveness transition. American Journal of Public Health, 93, 1261-1267.

Greason, P., \& Cashwell, C. (2009). Mindfulness and counseling self-efficacy: The mediating role of attention and empathy. Counselor Education and Supervision, 49, 2-19. doi:10.1002/j.1556-6978.2009.tb00083.x

Griffith, J. D., Rowan-Szal, G. A., Roark, R. R., \& Simpson, D. D. (2000). Contingency management in outpatient methadone treatment: a meta-analysis. Drug and Alcohol Dependence, 58, 55-66. doi:10.1016/S03768716(99)00068-X

Haug, N. A., Shopshire, M., Tajima, B., Gruber, V., \& Guydish, J. (2008). Adoption of evidence-based practices among substance abuse treatment providers. Journal of Drug Education, 38, 181-192. doi:10.2190/DE.38.2.f

Holm, S. (1979). A simple sequentially rejective multiple test procedure. Scandanavian Journal of Statistics, 6, 6570 . 
This is an author-produced, peer-reviewed version of this article. The final, definitive version of this document can be found online at Journal of Addictions \& Offender Counseling, published by American Counseling Association. Copyright restrictions may apply. doi: 10.1002/jaoc.12055

Iarussi, M. H., Tyler, J. M., Littlebear, S., \& Hinkle, M. S. (2013). Integrating motivational interviewing into a basic counseling skills course to enhance counseling self-efficacy. The Professional Counselor, 3, 161-174. doi:10.15241/mhi.3.3.161

Irvin, J. E., Bowers, C. A., Dunn, M. E., \& Wang, M. C. (1999). Efficacy of relapse prevention: a meta-analytic review. Journal of Consulting and Clinical Psychology, 67(4), 563-570. doi:10.1037/0022-006X.67.4.563

Kerwin, M. E., Walker-Smith, K., \& Kirby, K. C. (2006). Comparative analysis of state requirements for the training of substance abuse and mental health counselors. Journal of Substance Abuse Treatment, 30, 173181. doi:10.1016/j.jsat.2005.11.004

Kranz, K., \& O'Hare, T. (2011). Revalidating the substance abuse treatment self-efficacy scale (SATSES): A replication with social work practitioners. Journal of Social Work Practice in the Addictions, 11, 270-285. doi:10.1080/1533256X.2011.595290

Kranz, K. M. \& O'Hare, T. (2006). The Substance Abuse Treatment Self-Efficacy Scale. Journal of Social Service Research, 32, 109-121, doi:10.1300/J079v32n03_06

Kranz, K. (2003). Development of the alcohol and other drug self-efficacy scale. Research on Social Work Practice, 13, 724-741. doi:10.1177/1049731503254105

Larson, L. M., \& Daniels, J. A. (1998). Review of the counseling self-efficacy literature. The Counseling Psychologist, 26, 179-218. doi:10.1177/0011000098262001

Lee, T. K., Craig, S. E., Fetherson, B. T., \& Simpson, C. D. (2013). Addiction competencies in the 2009 CACREP clinical mental health counseling program standards. Journal of Addictions \& Offender Counseling, 34, 215. doi:10.1002/j.2161-1874.2013.00010.x

Lenz, A. S., Rosenbaum, L., \& Sheperis, D. (2016). Meta-analysis of randomized controlled trials of motivational enhancement therapy for reducing substance use. Journal of Addictions \& Offender Counseling, 37, 66-86. doi:10.1002/jaoc.12017

Lundahl, B., Moleni, T., Burke, B. L., Butters, R., Tollefson, D., Butler, C., \& Rollnick, S. (2013). Motivational interviewing in medical care settings: a systematic review and meta-analysis of randomized controlled trials. Patient Education and Counseling, 93, 157-168. doi:10.1016/j.pec.2013.07.012

Madson, M. B., Loignon, A. C., \& Lane, C. (2009). Training in motivational interviewing: A systematic review. Journal of Substance Abuse Treatment, 36, 101-109. doi:10.1016/j.jsat.2008.05.005

Martino, S., Ball, S. A., Nich, C., Canning-Ball, M., Rounsaville, B. J., \& Carroll, K. M. (2011). Teaching community program clinicians motivational interviewing using expert and train-the-trainer strategies. Addiction, 106, 428-441. doi:10.1111/j.1360-0443.2010.03135.x

McGovern, M. P., Fox, T. S., Xie, H., \& Drake, R. E. (2004). A survey of clinical practices and readiness to adopt evidence-based practices: Dissemination research in an addiction treatment system. Journal of Substance Abuse Treatment, 26, 305-312. doi:10.1016/j.jsat.2004.03.003

Miller, W. R., \& Rollnick, S. (2013). Motivational interviewing: Helping people change ( ${ }^{\text {rd }}$ Ed). New York, NY: Guilford Press.

Miller, W. R., Sorensen, J. L., Selzer, J. A., \& Brigham, G. S. (2006). Disseminating evidence-based practices in substance abuse treatment: A review with suggestions. Journal of Substance Abuse Treatment, 31, 25-39. doi:10.1016/j.jsat.2006.03.005

Moro, R. R., Wahesh, E., Likis-Werle, S. E., \& Smith, J. E. (2016). Addiction topics in counselor educator professional development: A content analysis. Journal of Addictions \& Offender Counseling, 37, 2-15. doi:10.1002/jaoc.12012

Moyer, A., Finney, J. W., Swearingen, C. E., \& Vergun, P. (2002). Brief interventions for alcohol problems: A meta-analytic review of controlled investigations in treatment-seeking and non-treatment-seeking populations. Addiction, 97, 279-292. doi:10.1046/j.1360-0443.2002.00018.x

Olmstead, T. A., Abraham, A. J., Martino, S., \& Roman, P. M. (2012). Counselor training in several evidence-based psychosocial addiction treatments in private US substance abuse treatment centers. Drug and Alcohol Dependence, 120, 149-154. doi:10.1016/j.drugalcdep.2011.07.017

Reese, R. J., Usher, E. L., Bowman, D. C., Norsworthy, L. A., Halstead, J. L., Rowlands, S. R., \& Chisholm, R. R. (2009). Using client feedback in psychotherapy training: An analysis of its influence on supervision and counselor self-efficacy. Training and Education in Professional Psychology, 3, 157-168. doi:10.1037/a0015673

Richardson, J.T.E. (2011). Eta squared and partial eta squared as measurements of effect size in educational research. Educational Research Review, 6, 135-147. doi:10.1016/j.edurev.2010.12.001 
This is an author-produced, peer-reviewed version of this article. The final, definitive version of this document can be found online at Journal of Addictions \& Offender Counseling, published by American Counseling Association. Copyright restrictions may apply. doi: 10.1002/jaoc.12055

Riper, H., Andersson, G., Hunter, S. B., de Wit, J., Berking, M. and Cuijpers, P. (2014), Treatment of comorbid alcohol use disorders and depression with cognitive-behavioural therapy and motivational interviewing: a meta-analysis. Addiction, 109, 394-406. doi:10.1111/add.12441

Sayegh, C. S., Huey, S. J., Jr., Zara, E. J., \& Jhaveri, K. (2017). Follow-up treatment effects of contingency management and motivational interviewing on substance use: A meta-analysis. Psychology of Addictive Behaviors, 31, 403-414. doi:10.1037/adb0000277

Schiele, B. E., Weist, M. D., Youngstrom, E. A., Stephan, S. H., \& Lever, N. A. (2014). Counseling self-efficacy, quality of services, and knowledge of evidence-based practices in school mental health. The Professional Counselor, 4, 467-480. doi:10.15241/bes.4.5.467

Schumacher, J. A., Madson, M. B., \& Norquist, G. (2011). Using telehealth technology to enhance motivational interviewing training for rural substance abuse treatment providers: A services improvement project. The Behavior Therapist, 34, 64-70.

Schwalbe, C. S., Oh, H. Y., \& Zweben, A. (2014). Sustaining motivational interviewing: A meta-analysis of training studies. Addiction, 109, 1287-1294. doi:10.1111/add.12558

Singal, A. G., Higgins, P. D., \& Waljee, A. K. (2014). A primer on effectiveness and efficacy trials. Clinical and Translational Gastroenterology, 5(1), e45.

Substance Abuse and Mental Health Services Administration (2017). Behavioral Health Barometer: United States, Volume 4 (HHS Publication No. SMA-17-BaroUS-16). Rockville, MD. Retrieved from https://store.samhsa.gov/shin/content/SMA17-BAROUS-16/SMA17-BAROUS-16.pdf

Urbani, S., Smith, M. R., Maddux, C. D., Smaby, M. H., Torres-Rivera, E., \& Crews, J. (2002). Skills-based training and counseling self-efficacy. Counselor Education and Supervision, 42, 92-106. doi:10.1002/j.15566978.2002. tb01802.x

Wright, S. P. (1992). Adjusted p-values for simultaneous inference. Biometrics, 48, 1005-1013.

U.S. Bureau of Labor Statistics, United States Department of Labor. (2015). Occupational Outlook Handbook, 2014-2015. Retrieved from http://www.bls.gov/ooh/community-and-social-service/substance-abuse-andbehavioral-disorder-counselors.htm

VanBuskirk, K. A., \& Wetherell, J. L. (2014). Motivational interviewing with primary care populations: a systematic review and meta-analysis. Journal of Behavioral Medicine, 37, 768-780. doi:10.1007/s10865013-9527-4

Vasilaki, E. I., Hosier, S. G., \& Cox, W. M. (2006). The efficacy of motivational interviewing as a brief intervention for excessive drinking: A meta-analytic review. Alcohol and Alcoholism, 41, 328-335. doi:10.1093/alcalc/agl016

Weissman, M. M., Verdeli, H., Gameroff, M. J., Bledsoe, S. E., Betts, K., Mufson, L., ... \& Wickramaratne, P. (2006). National survey of psychotherapy training in psychiatry, psychology, and social work. Archives of General Psychiatry, 63, 925-934. doi:10.1001/archpsyc.63.8.925 
This is an author-produced, peer-reviewed version of this article. The final, definitive version of this document can be found online at Journal of Addictions \& Offender Counseling, published by American Counseling Association. Copyright restrictions may apply. doi: 10.1002/jaoc.12055

Table 1

Means, Standard Deviations, and Contrast for Counselor Self-Efficacy

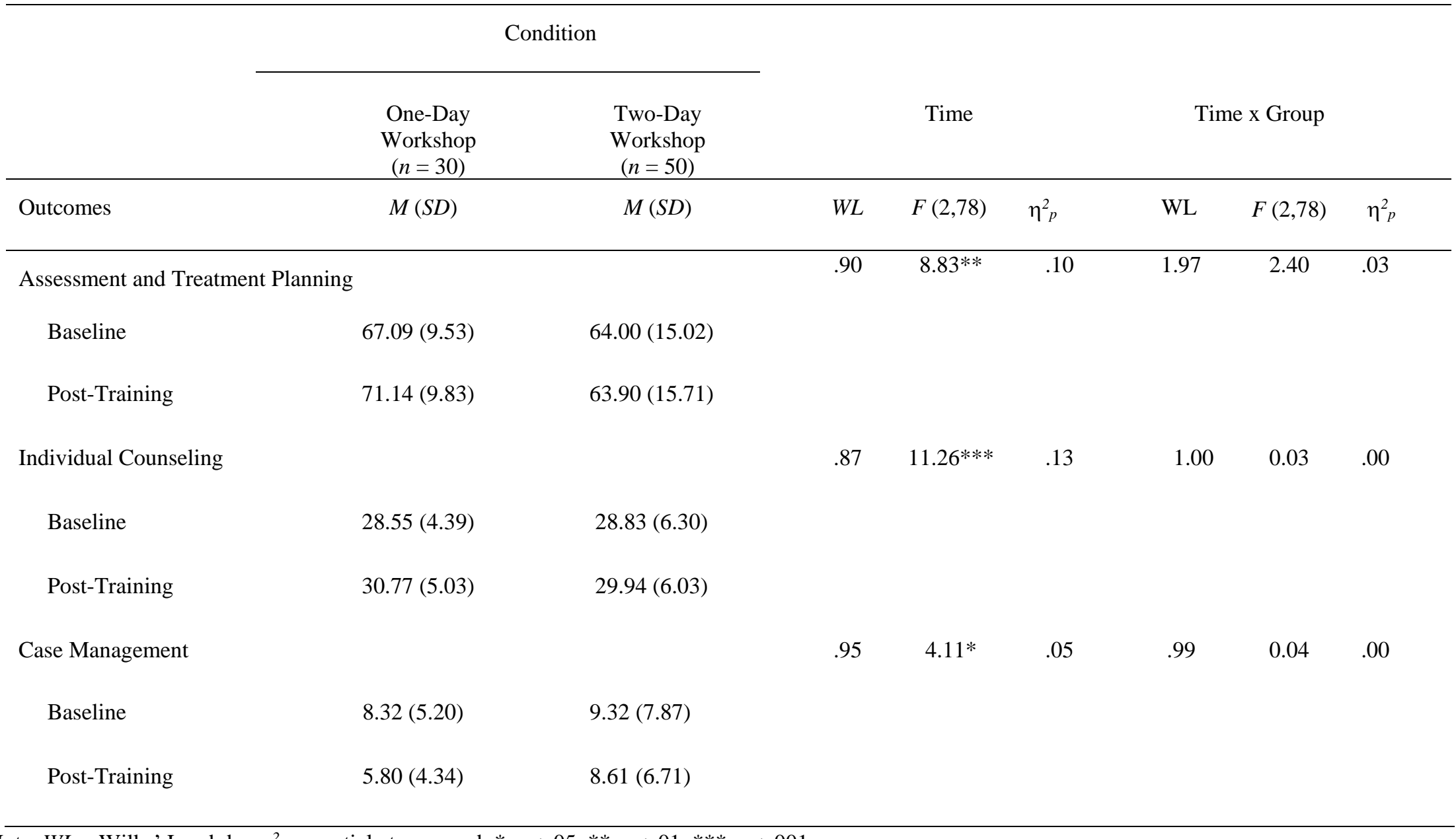

Note. $W L=$ Wilks' Lambda; $\eta_{p}^{2}=$ partial eta squared; $* p<.05, * * p<.01, * * * p<.001$. 